\title{
Smart Internet of Isolated Capacitor Trip Device for High-voltage Protective Relay of Backup Power
}

\author{
Cheng-Tsung Huang, ${ }^{1}$ Ho-Chi Lien, ${ }^{2}$ Chi-Pan Lin, ${ }^{1}$ and Wen-Ping Chen ${ }^{*}$ \\ ${ }^{1}$ Department of Electrical Engineering, National Kaohsiung University of Science and Technology, \\ No. 415, Jiangong Rd., Sanmin Dist., Kaohsiung City 80778, Taiwan, Republic of China \\ ${ }^{2}$ Department of Fire Science, Tajen University, \\ No. 20, Weixin Rd., Yanpu Township, Pingtung County 90741, Taiwan, R.O.C.
}

(Received December 5, 2019; accepted July 28, 2020)

Keywords: capacitor trip device, self-test, real-time notification, protective relay

In high-voltage power systems, overload or short-circuit accidents occur frequently owing to the failure of the user equipment, leading to the occurrence of power accidents. Therefore, protective relays must be installed before and after each feeder to isolate the occurrence of faults and thus reduce damage to the power system. However, when the working power supply of the protective relay loses power, it will not be possible to isolate the accident feeder, and the failure of the power system will expand, severely affecting the reliability of the system power supply. Therefore, it is expressly stated by the Taiwan Power Company that a capacitor trip device (CTD) must be installed to ensure a power supply to the protective relay for highvoltage users. However, CTDs currently on the market often fail to ensure the proper operation of the protective relay owing to an insufficient capacitance or a low output operating voltage. Therefore, we propose a high-capacity CTD capable of performing self-tests and instant notification for network monitoring. Compared with the conventional CTD, our CTD can prevent instantaneous large current transfer to the powered device, ensuring that the power supply of the powered device is uninterrupted. It can accomplish the real-time monitoring of the power status, provide a stable power supply, prevent overcurrent, and protect equipment. It is a very important contribution to the protection of the relay system.

\section{Introduction}

The discovery and popularity of electricity have brought about the unprecedented development of scientific and technological civilization in human history, and electric power has become an indispensable element in people's lives. Moreover, with the improvement of human living standards, people become increasingly dependent on the stable supply of electricity. After the invention of the transformer and its commercialization as a power transformer by Westinghouse Electric in the United States in 1885, the power transmission efficiency of the electric power system increased in a high-voltage and low-current manner, enabling the transmission of electric energy to distant users. Accordingly, large-scale power "Corresponding author: e-mail: wpc@nkust.edu.tw https://doi.org/10.18494/SAM.2020.2782 
plants became more beneficial in terms of cost performance, and the electricity produced could also be transmitted to places a long distance away. However, since electricity cannot be efficiently stored continuously in large amounts for back-up use, power companies must produce sufficient electricity for users and ensure the continuity and quality of high-voltage power systems. In addition, power companies must reserve a portion of the power generation capacity to satisfy emergency power demand, giving the power system full flexibility to cope with irregular issues, such as extreme weather, machine failure, and fuel shortage. The emergence of the electric power system has promoted changes in many fields of social production and launched the electric power era, thereby prompting the second technological revolution in the history of modern times (the so-called Industry 2.0). The degree of development and technical level of power systems have become indicators of the economic development of countries. The extensive application of the electric power system has instigated the ever-changing technological development of human beings, and all aspects of the basic necessities of life, education, and entertainment are closely tied to electricity. The centralized and unified distribution of electricity by power systems tightly bonded between power supply and consumption. The integrated power system undertakes the production and distribution of electric energy, wherein the production, supply, distribution, and consumption of electricity are carried out simultaneously. Hence, in the production of the power system, the continuity and balance of the power supply must be ensured. To prevent the instability of the power system caused by abnormal power consumption accidents at the user end, the protective relay of power feeder lines plays an important role in cutting off the fault. ${ }^{(1,2)}$

Electricity faults are usually caused by high-voltage power supply, most of which result from overload or short circuit in the high-voltage system, and ultimately lead to electric accidents. If the working power of the trip circuit fails, the protection relay is not activated; therefore, the main protective circuit breaker receives no signals to trigger tripping or the circuit breaker fails to receive power for tripping and accidents caused by electric faults will worsen, thus seriously affecting the reliability of the power system supply. Therefore, in terms of supplying working power to the protection relay of high-voltage users, Taiwan Electric Power Company stipulated in 2003 that a capacitor tripping device (CTD) must be equipped to ensure the operation of the power system supply. However, the malfunction of protection relays is a result of the insufficient capacitance or low output operating voltage of the CTDs currently on the market. With the worsening of the global greenhouse effect and frequent accidents at nuclear power plants, countries around the world have attached importance to the use of green energy, namely, renewable energy sources, for power generation. ${ }^{(3,4)}$ For example, Taiwan Electric Power Company initially estimated that Taiwan's land-based wind power can be used to generate 1.2 GW of electricity, and offshore wind may provide $6.2 \mathrm{GW}$. In addition, according to data from the Energy Ministry, Taiwan's solar photovoltaic power can theoretically be as high as 35 GW. As a result, research on renewable energy such as wind power ${ }^{(5-7)}$ and solar power ${ }^{(8-10)}$ and their development have gradually attracted the attention of countries around the world. Green energy technology has become the direction of the energy development of national governments of advanced nations. It is critical to protect the power between each feeder line and high-voltage users in the power system to solve the problem of unstable power generation 
of green energy sources, ${ }^{(11)}$ so ensuring the operation of protective relay stations is the top priority. ${ }^{(12-14)}$ Particularly in high-voltage power systems, overload or short-circuit issues originating from user equipment result in frequent power accidents. Therefore, a protective relay must be installed at the front and rear of each feeder line to isolate faults and minimize harm to the power system. Nevertheless, when the working power of the protective relay is disabled, the feeder in the event of an accident cannot be isolated, resulting in the worsening of power system faults and serious effects on the reliability of the system power supply. Therefore, as stated above, CTDs must be equipped to ensure the operation of the power system supply. ${ }^{(15,16)}$ However, the malfunction of relays due to the insufficient capacitance or low output operating voltage of the CTDs currently on the market renders an isolated capacitor tripping device with the functions of self-detection and instant fault notification, one of the most important products in protective circuits of power systems. Fortunately, the advancement of sensing and network technologies has set off the industry 4.0 wave, and technologies with self-awareness and remote control have begun to be applied to important equipment of power systems, such as a smart Internet of a transformer, which is one of the developed applications. ${ }^{(17,18)}$ In this paper, we propose a smart Internet of the isolated capacitor trip device (Smart-CTD). The technical content of Smart-CTD development mainly includes the following: software and hardware technological designs in the current-limiting protection circuit, the rectification and voltage stabilization circuit, transmission communication, and the human and machine interface (HMI) of computers controlled by supervisory control and data acquisition (SCADA). In addition, the power measurement circuit includes a transmission communication module; the computer controlled by SCADA can measure and report the power status in real time through the transmission communication module and prevent excessive current from damaging high-voltage electrical equipment through cooperation between the thermoelectric resistor and the fuse. Compared with common CTD products, Smart-CTDs can prevent instantaneous excessive current from being transmitted to the electrical equipment, thus ensuring the continuity of its power supply, measure and report the power status in real time, stabilize the power supply, prevent overcurrent, and protect equipment from being damaged.

\section{Smart-CTD}

In this paper, we proposed a Smart-CTD with the capability of self-test and instant notification of faults for application in high-voltage power supply system equipment, as shown in Fig. 1. In the overall system architecture, the SCADA central control computer with HMI converts the USB communication transmission into RS-485 two-way control signals through a signal converter, thereby remotely monitoring the entire Smart-CTD system. In addition, the Smart-CTD can also be built through the web platform of the SCADA central control computer. Users can remotely monitor the entire Smart-CTD system through a browser in their mobile phone, tablet or remote computer. Furthermore, using microcontrol unit (MCU) communication chips, in addition to determining whether the remote central control computer has communication data, the Smart-CTD usually monitors the AC voltage (primary) and the DC voltage (secondary) and independently determines whether the voltage is normal. If an 


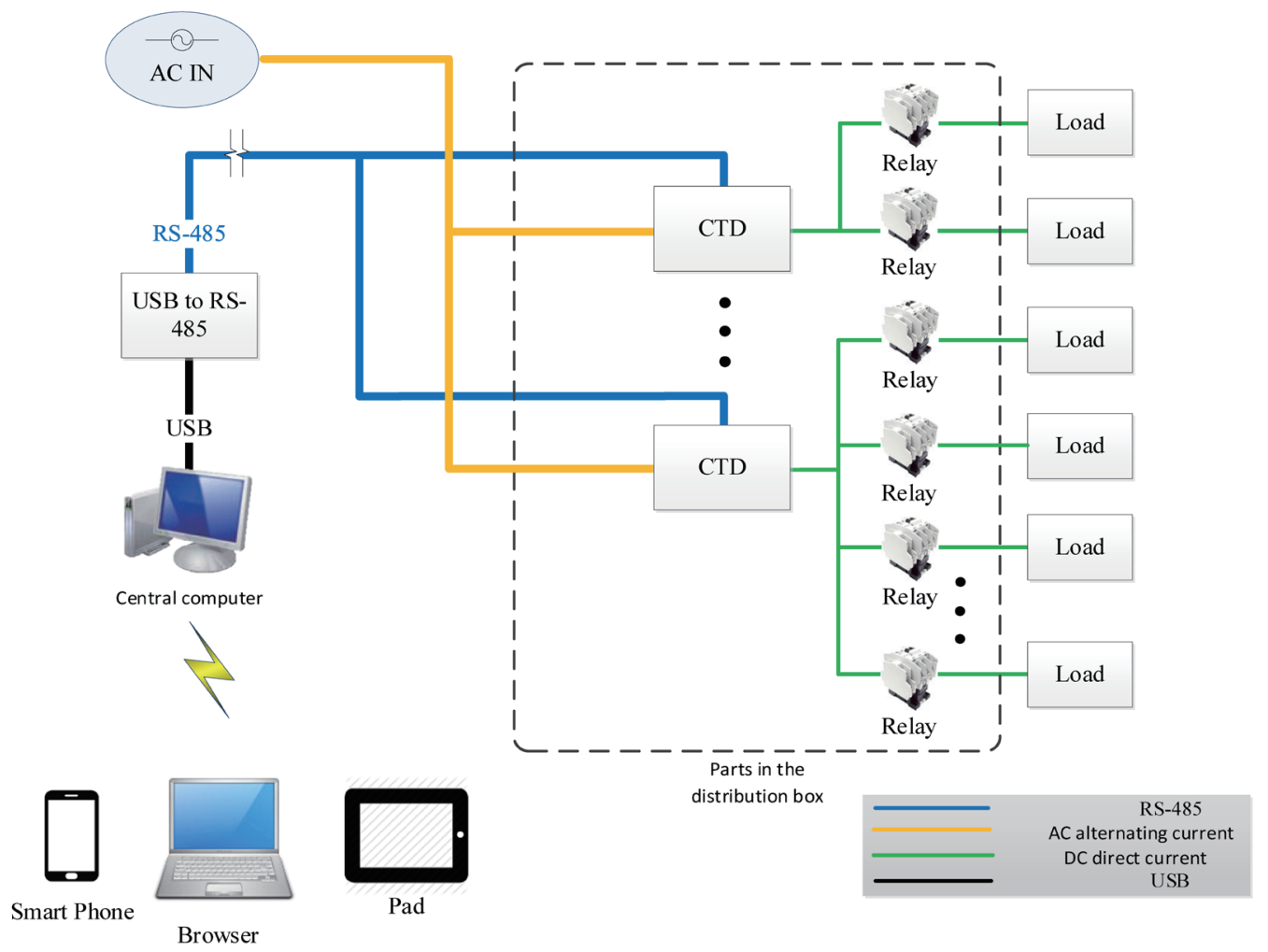

Fig. 1. (Color online) Structure of developed Smart-CTD system.

abnormality occurs, it initiates the action to output dry contact and reports the communication abnormality outward.

The Smart-CTD includes a multifunctional isolated capacitor trip device (multifunctional CTD) and the remote monitoring communication system. The former comprises the DC power supply system, the self-aware detection system, and the transmission communication system, while the latter is required by the SCADA control computer monitoring system. The detailed description of each unit is as follows.

\subsection{Multifunctional CTD}

The system architecture of the Smart-CTD is shown in Fig. 2, in which the internal circuit block of the multifunctional CTD includes four parts: a bridge full-wave rectification and stabilization circuit, a current-limit protection circuit, a power storage module, and a perceptual detection system. The multifunctional CTD provides hardware technologies, such as overcurrent protection, DC output voltage and detection of abnormal capacitance compared with that of the ordinary CTD, as well as communication technologies of the real-time transmission of software technology faults, enabling the user's SCADA monitoring system to grasp the capacitance status and AC/DC voltage working status of all installed multifunctional CTDs in real time and to predict faults of the multifunctional CTD before their occurrence. Moreover, the SCADA monitoring system can also utilize the digital output (DO) alarm contact 


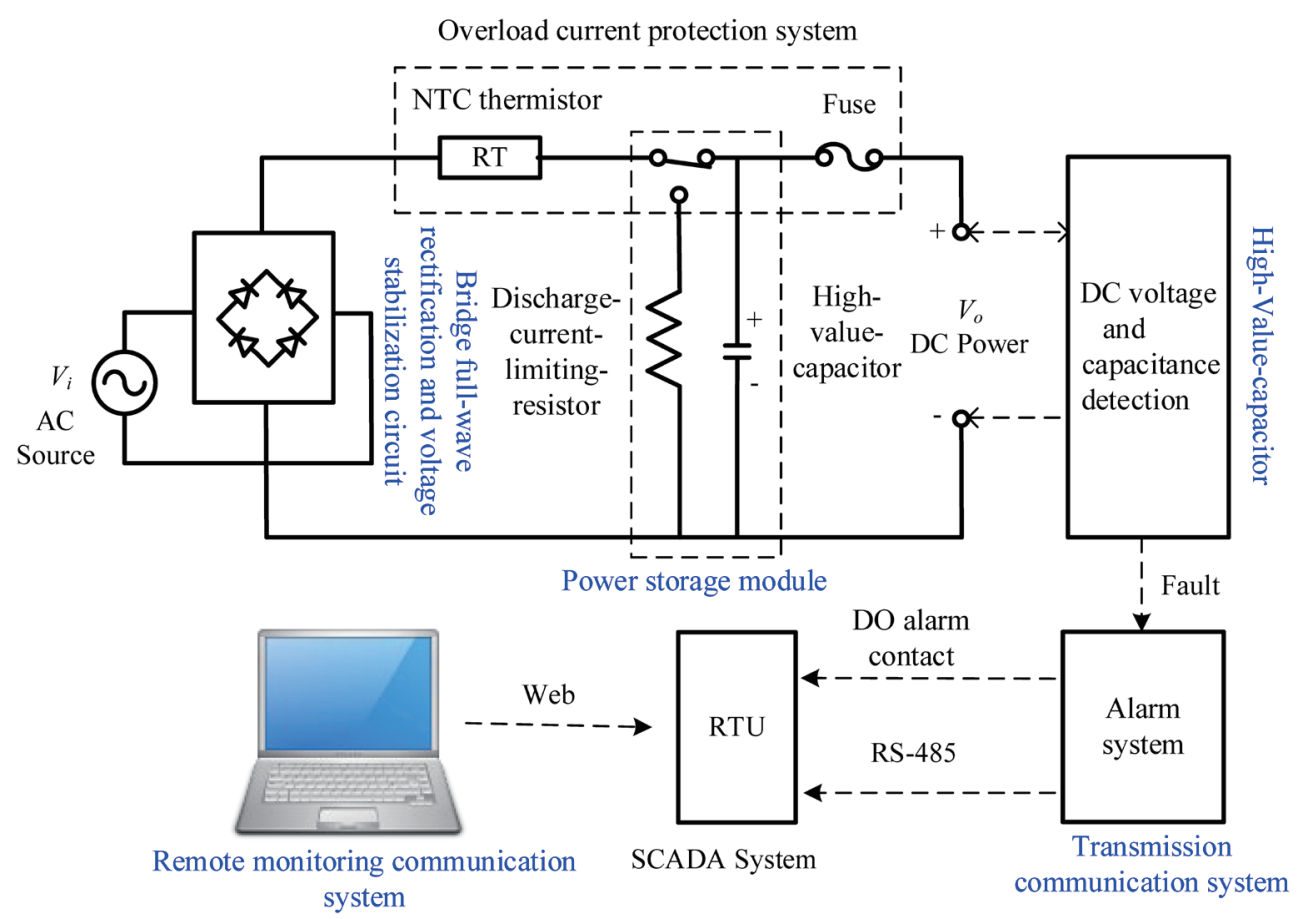

Fig. 2. (Color online) Circuit design architecture of Smart-CTD.

of the functional CTD to grasp the capacitance status and AC/DC voltage working status of the multifunctional CTD itself.

(1) Bridge full-wave rectification and stabilization circuit (as shown in Fig. 2): A full-wave rectification circuit design is adopted to transform the mains voltage using the turn ratio of the isolation transformer for voltage reduction to obtain sine waves of two sets of winding voltage. When the original input sine wave $(\sin w t)$ of the rectifier circuit is $V_{i}=V_{m}$, where $V_{m}$ is the peak or max voltage of a waveform, the average of the full-wave pulsating DC voltage is $V_{d c}=2 / \pi V_{m}$. If the output voltage requirement is $V_{d c}=125 \mathrm{~V}$, then one test voltage is $V_{i}=80 \mathrm{~V}$. In the design of the trip circuit, when the primary voltage input is interrupted, the secondary output voltage is switched to the capacitor-powered output.

(2) Current-limiting protection circuit: Overcurrent protection and fuse protection were added to the DC circuit of the CTD. The latter was achieved by adding a fuse to the DC power supply load of the CTD for overload protection. The former is a protection element with a negative-temperature-coefficient (NTC) thermistor installed after the rectified AC end of the CTD, because tandem connection to the NTC thermistor in the circuit can suppress the inrush current at startup. After the suppression of the inrush current, the resistance of the NTC thermistor drops to a very small value and the power consumption becomes negligible. Therefore, the CTD can automatically reduce the current when the operating temperature of the device becomes too high to prevent excessive current from damaging the appliance. That is, when the temperature is very high and the current can no longer be reduced, the line for 
supplying electric power will be disconnected to protect the back-end electrical appliances from being burned. The addition of the NTC thermistor and fuse to the multifunctional CTD prevents excessive current from damaging the hardware of the multifunctional CTD itself and avoids overburning by the generated instantaneous high current, thereby averting the interruption of the power supply to the back-end protection equipment of the multifunctional CTD.

(3) Power storage module: The DC output power of the CTD is generally required to be $125 \mathrm{~V}$, and the discharge time of the capacitor value needs to be 3-5 s. In this study, we used highvalue capacitors $(10000 \mu \mathrm{F})$ to meet the required discharge time of more than 5 to $10 \mathrm{~s}$ for the multifunctional CTD and added a function to test DC discharge, that is, when the switch at the $\mathrm{AC}$ power supply was opened to discharge the high-value capacitor, a current-limiting resistor was set to limit the discharge current to ensure safe discharge.

(4) Perceptual detection system: it is mainly used to detect whether the capacitance value of the CTD and the DC output voltage are abnormal. Its technology includes calibration circuit designs for $\mathrm{AC}$ and $\mathrm{DC}$, for the primary-side $\mathrm{AC}$ voltage detection circuit, and for the secondary-side capacitor voltage detection circuit. The details are as follows.

(a) Design of $\mathrm{AC}$ calibration circuit: Figure 3 shows the $\mathrm{AC}$ voltage detection circuit. The mains sampling circuit uses a resistance voltage dividing method, for which the voltageresistance specification must be considered when being designed. Thus, a high-voltage resistor is used to prevent insufficient voltage resistance. The sampling point is from the $\mathrm{AC}$ capacitor, and to prevent the interference of the feedback signal, the attenuated signal avoids other high-voltage signals and high-frequency switching signals on the power board, thereby causing abnormal feedback. After the voltage is divided, it is distributed by the operational amplifier (OPA) differential sampling circuit and its signal will be processed by a set of simple RC low-pass filter and two-group 1N4148 over-voltage protection circuit before entering the DSP. The design of the RC low-pass filter circuit requires high-frequency noise filtering above $50 / 60 \mathrm{~Hz}$ to prevent noise interference. However, because of the generation of a negative voltage signal, the feedback signal

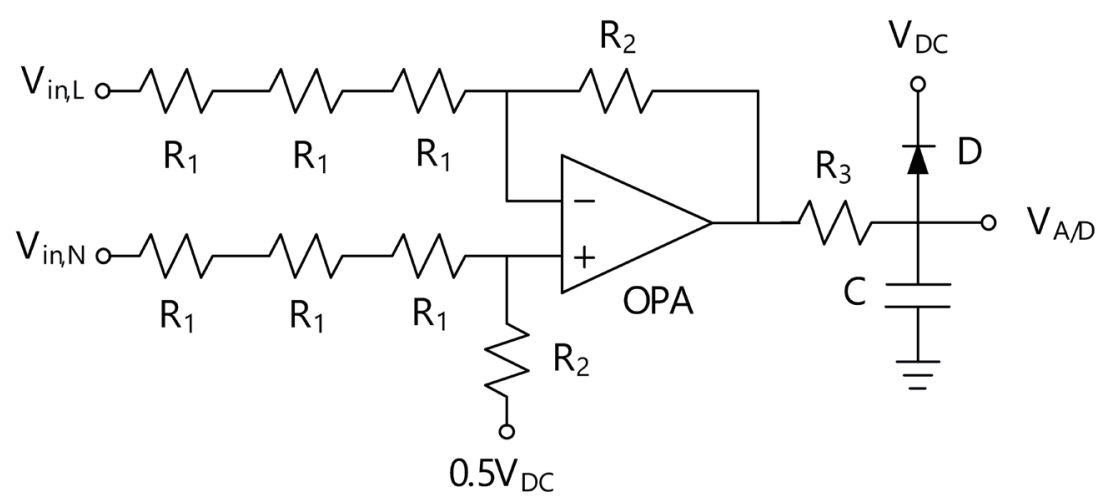

Fig. 3. Design diagram of the voltage detection circuit. 
cannot be provided directly to the MCU. Hence, after the full-wave rectification of the mains voltage signal, the potential must be controlled at a positive voltage level before it can be used by the MCU, but the full-wave rectification will cause signal distortion due to the decrease in the conduction voltage of diodes, thereby resulting in feedback signal error. Therefore, in this design, $+1.65 \mathrm{~V}$ voltage correction is added to increase the OPA feedback signal level to $+1.65 \mathrm{~V}$ to solve the problem of the negative voltage signal directly entering the pin of the MCU A/D. The ratio formula of power conversion shown in Fig. 3 is as follows, where $V_{D C}=3.3 \mathrm{~V}, R_{1}=300 \mathrm{k} \Omega, R_{2}=3.9 \mathrm{k} \Omega, R_{3}=1.0 \mathrm{k} \Omega, C=1$ $\mu \mathrm{F}$, and $D$ is the IN 4001 diode.

$$
V_{A / D}=\left(V_{i n, L}-V_{i n, N}\right) \times \frac{R_{2}}{R_{1}+R_{1}+R_{1}}+V_{D C 2}
$$

(b) Design of DC calibration circuit: The design of the DC calibration circuit is shown in Fig. 4. When the mains sine wave is detected by the A/D of the CPU, since the mains sine wave has positive and negative piezoelectric potentials, the use of OPA with a single power supply will result in a negative voltage problem in the feedback signal. Therefore, during the design, the feedback signal level will be increased to half the power usually used by the MCU, namely, $+1.65 \mathrm{~V}$ [as shown in Eq. (2)], to solve the problem of negative voltage signals entering the digital signal processor (DSP). The easiest method of implementing this design is by the resistance voltage dividing method. In this design, the routing path must be closest to the power supply of the MCU, and double R is installed for voltage division to capture half the voltage. However, because of the load effect of the resistance voltage dividing method, in order to improve the load effect, the divided power supply will pass through the OPA follower and then be used as the reference potential of the OPA circuit. The power conversion ratio is shown below, where $V_{\text {offset }}$ is the feedback signal level, $V_{D C}=3.3 \mathrm{~V}, R=26 \mathrm{k} \Omega, C_{1}=1 \mu \mathrm{F}$, and $C_{1}=10 \mu \mathrm{F} / 50 \mathrm{~V}$.

$$
V_{\text {offset }}=V_{D C} \times \frac{R}{R+R}=+1.65 \mathrm{~V}
$$

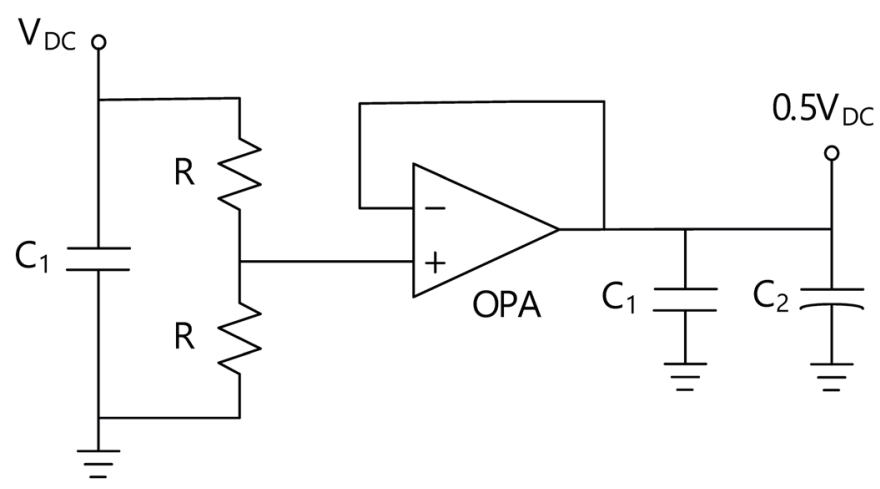

Fig. 4. DC calibration circuit design. 
The firmware programming flowchart of the proposed detection system is shown in Fig. 5. First, obtain the AC voltage on the primary side by using the power measurement circuit, as well as the correct value based on magnification calculation. Then, obtain the secondary DC voltage and the correct value based on magnification calculation. Next, determine whether there is a communication signal from the transmission communication module; if not, determine whether the switch is on, or else, determine whether the data is correct. The determination is based on the cyclic redundancy check (CRC) inspection, which will be explained in detail later. If the data is correct, the process will follow the signal command and send the monitored value signal.

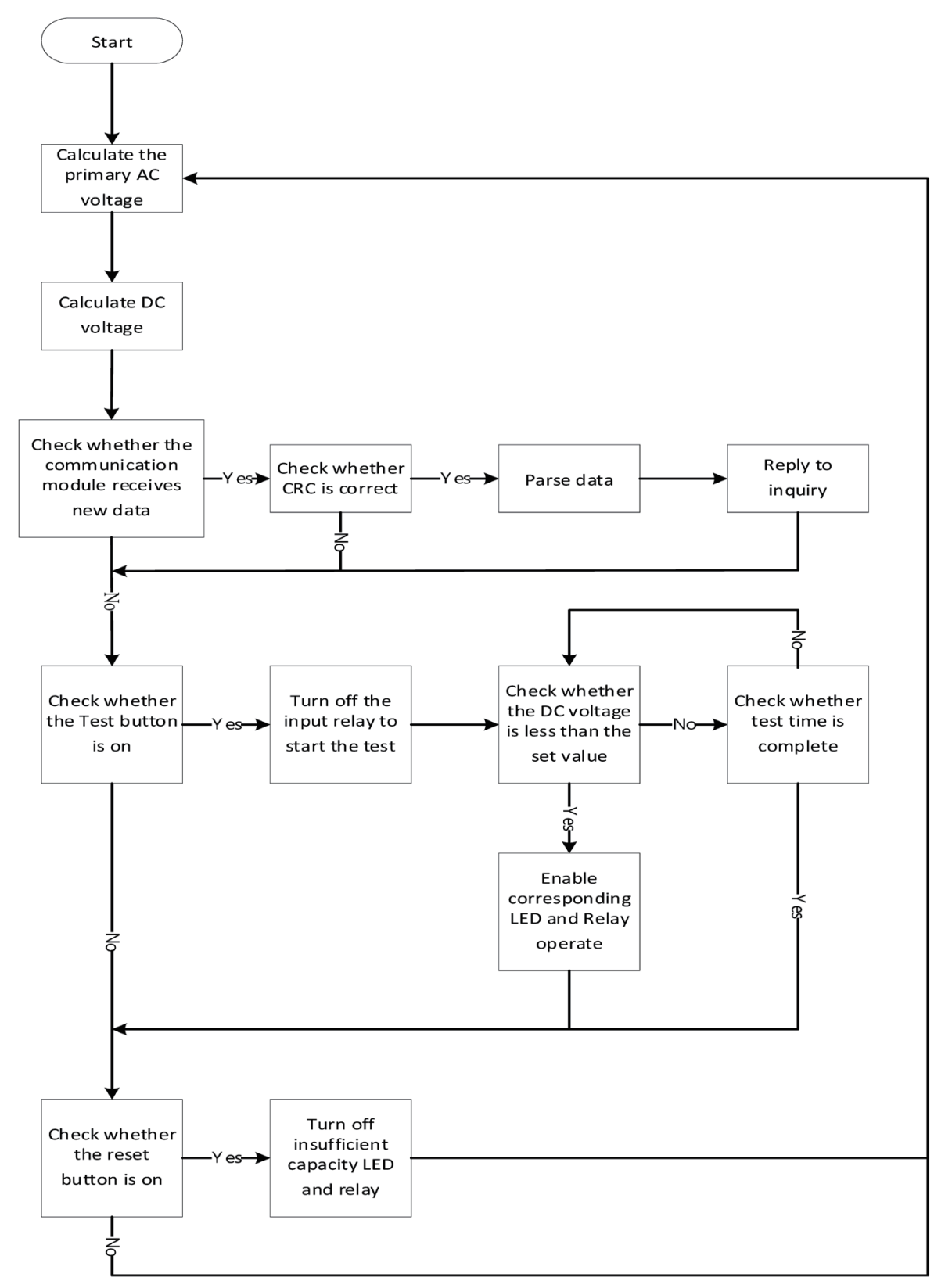

Fig. 5. Firmware programming flowchart. 
(5) Transmission communication system: The multifunctional CTD transmission communication technology uses RS-485 communication, as shown in Fig. 6. RS-485 is a standard specified by power characteristics belonging to the physical layer of the OSI model, which includes 2-wire, half-duplex, and multipoint communication. Its power characteristics are very different from those of RS-232. The voltage difference across the cable represents the transmitted signal; the voltage of 1 pole is identified as logic 1 and that of the other is identified as logic 0 . This is valid when the voltage difference between the two ends is at least $0.2 \mathrm{~V}$, and any difference between -7 and $12 \mathrm{~V}$ is considered correct for the receiving end. RS-485 only specifies the power characteristics of the receiving and transmitting ends without specifying or recommending any information agreements. RS-485 can be applied to configure an inexpensive WAN and use communication links of "one sender + multireceivers". In addition, it provides high-speed data communication (35 Mbit/s at $10 \mathrm{~m} ; 100 \mathrm{kbit} / \mathrm{s} 1200 \mathrm{~m}$ ). RS-485 and RS-422, which use a twisted pair cable for highvoltage differential balanced transmission, are applicable to long-distance and largearea transmission (more than 4000 feet, $1200 \mathrm{~m}$ ). In contrast to RS-422, which uses a nonconvertible single sender, the sender of RS-485 must be set to the send mode, which enables RS-485 to adopt the two-wire mode to implement multipoint and bidirectional communication.

RS-485 is recommended to be used in point-to-point, line-type, and bus-type networks, rather than in star or ring networks. Ideally, RS-485 requires two termination resistors, the resistance of which must be equal to the characteristic impedance of the transmission cable. Without the characteristic impedance, noise will occur when all devices are stationary or without energy, and the line shift requires a voltage difference at both ends. Without a termination resistor, the faster transmitting end will generate multiple data signal edges, some of which are incorrect. The reason why a star or ring topology cannot be used is that they have unnecessary reflections. Too low or too high a termination resistance can generate

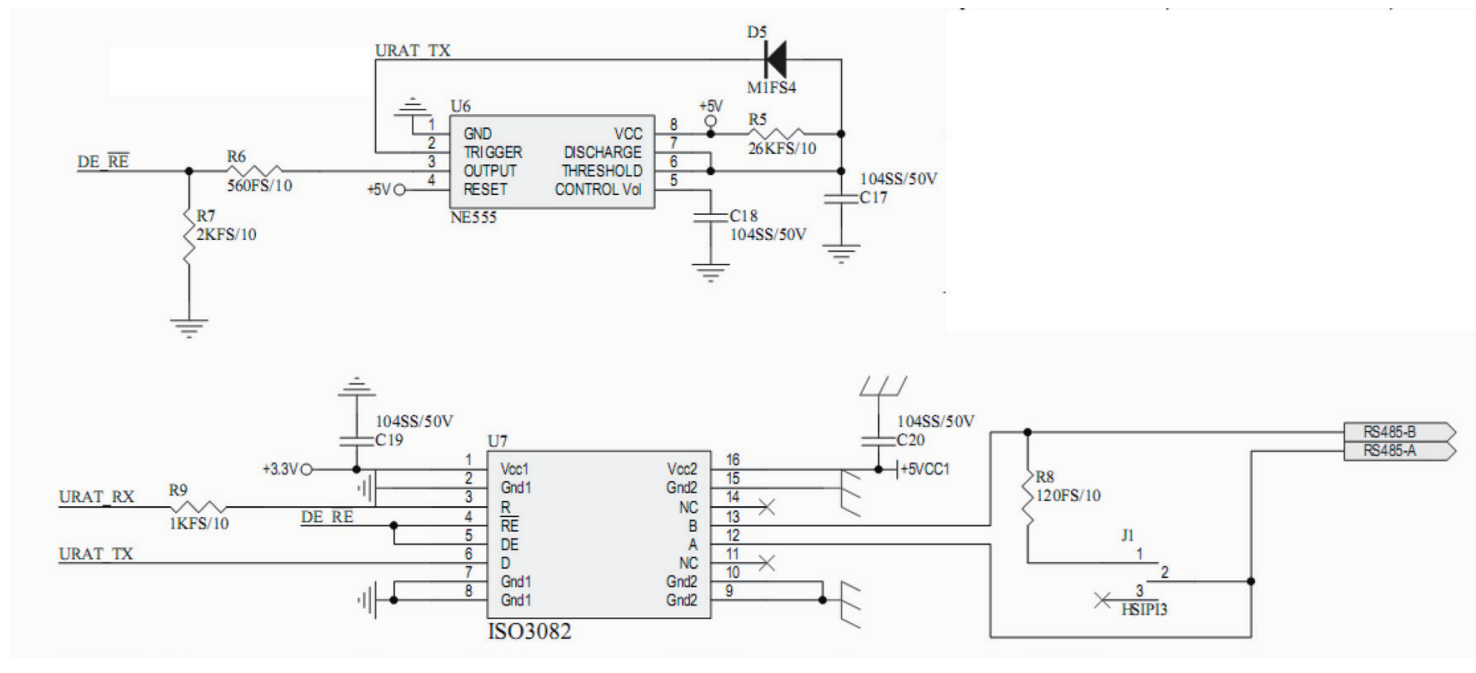

Fig. 6. Diagram of RS-485 communication circuit. 
electromagnetic interference. RS-485 can implement full duplex transmission similarly to RS-422 when using four wires, RS-485 can implement true multipoint communication, and RS-485 and RS-422 can implement mutual connection under certain restrictions.

(1) Communication interface and specifications of the remote monitoring communication system, the switch device, and the power distribution control device:

(a) RS-485 (1.2 km distance), (b) the communication protocol is customized,

(c) baud rate: 9600 ,

(d) parity check: $\mathrm{N}$,

(e) data bits: 8 bits,

(f) stop bits: 1 bit.

(2) Communication protocol: This system uses the Modbus remote terminal unit (RTU) communication format, the communication protocol formats of which are shown in Table 1. The format includes the device ID, baud rate, AC voltage (primary), DC voltage (secondary), as well as the communicable transmission capacity status and voltage status.

\subsection{Remote monitoring communication system}

When the high-voltage equipment power system has many multifunctional CTD monitoring devices, disturbances in the operation and management easily arise. To effectively manage and monitor the multifunctional CTD, the monitoring system plans a man-machine interface that can quickly present the current status of the system. In this interface, the control personnel can also quickly and clearly understand the actual operation of each device to ensure a quick and accurate elimination of abnormalities. The remote monitoring communication system uses RS-485 serial transmission in line with the Modbus RTU format communication protocol and verifies the correctness of the data by the CRC 16 method. Therefore, the design of the remote monitoring communication system, design methods of RS-485 serial transmission, and the communication protocol are expounded here. The functions of the system are divided into three major blocks, as shown in Fig. 7, namely, system management, monitoring operations, and report presentation. Related specifications are as follows.

(a) System management: In terms of the security and maintenance of the entire system, the system will execute permission control for users. The flowchart is shown in Fig. 8. When inputting the correct account and password, users can smoothly enter the system for operation, and the system will also give the corresponding interface for operation in

Table 1

Protocol formats.

\begin{tabular}{|c|c|c|c|}
\hline \multicolumn{4}{|c|}{ Modbus RTU Mode Protocol Address Map } \\
\hline Location & Name & Specifications & Action \\
\hline 0000 & Address (ID) & Communication location, input range $0000-00 \mathrm{FF}(0-255)$ & $\mathrm{R} / \mathrm{W}$ \\
\hline 0001 & Baud Rate & Baud rate, input 0001:19200, 0002:9600, 0003:4800, 0004:2400 & $\mathrm{R} / \mathrm{W}$ \\
\hline 0002 & AC Voltage & AC voltage on the primary side & $\mathrm{R}$ \\
\hline 0003 & DC Voltage & DC voltage on the secondary side & $\mathrm{R}$ \\
\hline 0004 & Capacity Status & $\begin{array}{l}\text { Capacity status, a value of } 0001 \text { indicates insufficient capacity warning; } \\
\text { a value of } 0000 \text { indicates normal }\end{array}$ & $\mathrm{R}$ \\
\hline 0005 & Voltage Status & $\begin{array}{l}\text { Voltage status, a value of } 0001 \text { indicates insufficient voltage warning; } \\
\text { a value of } 0000 \text { indicates normal }\end{array}$ & $\mathrm{R}$ \\
\hline
\end{tabular}




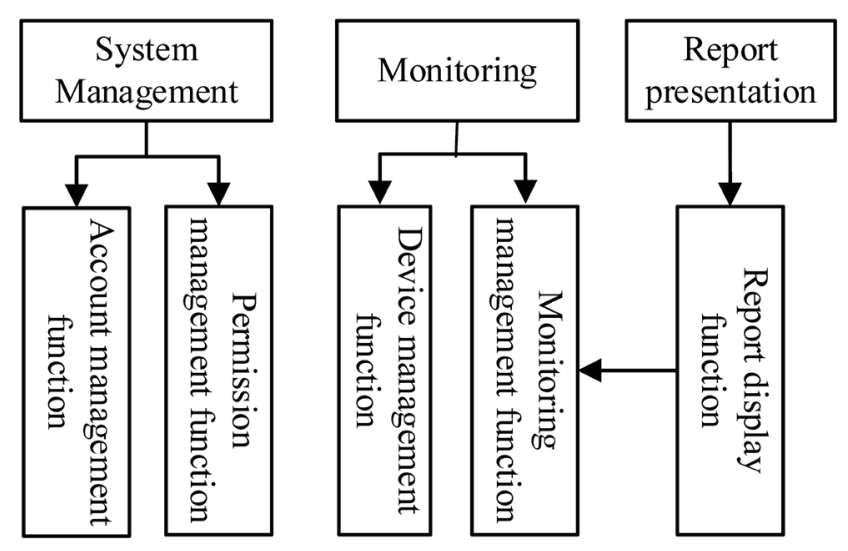

Fig. 7. Architecture of the remote monitoring communication system.

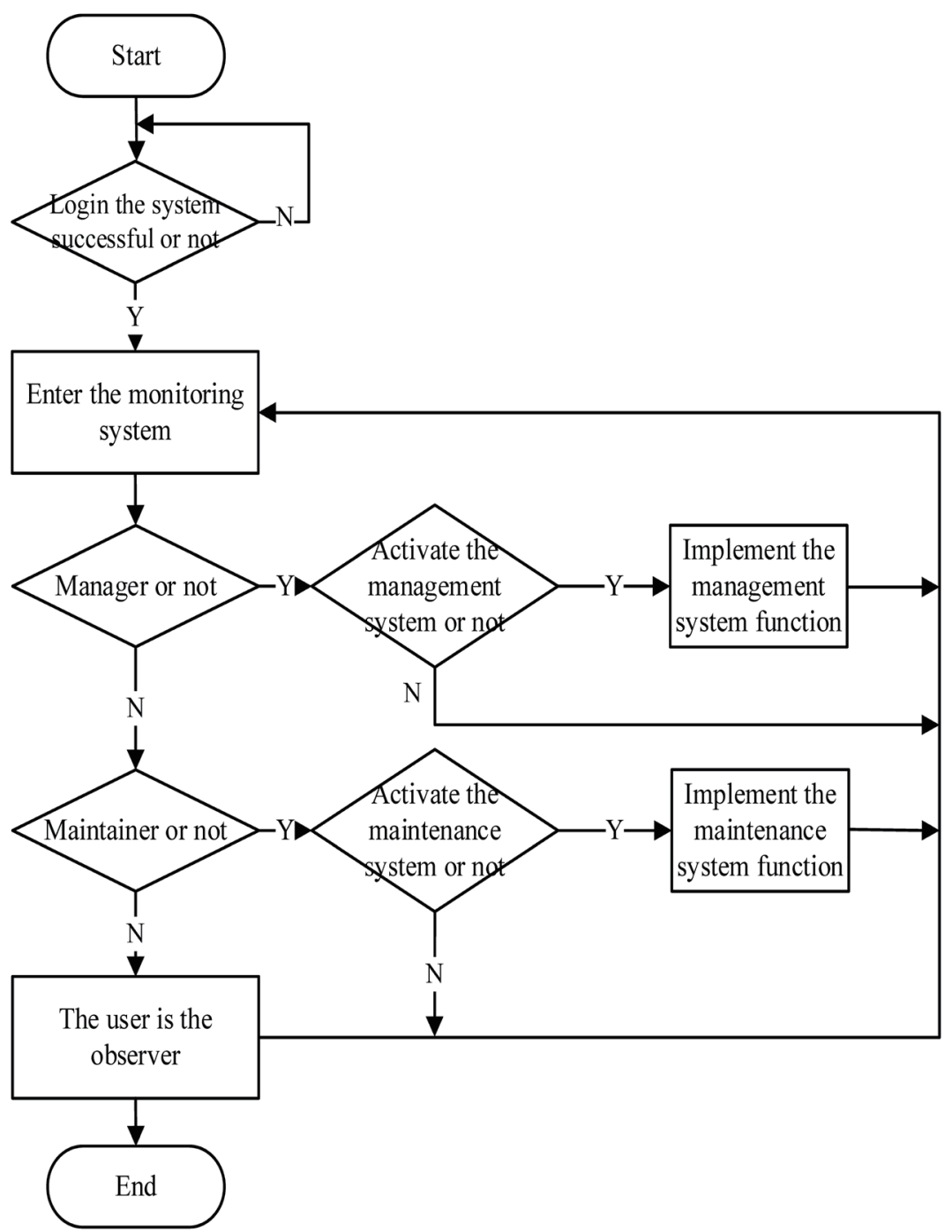

Fig. 8. Flowchart of system management. 
accordance with the user's authority. The user with the highest authority is defined as the administrator. Logging into the system enables the administrator to set permissions for users. When the equipment needs to be expanded or downsized, the software can be reconfigured accordingly, so that the system can operate more efficiently. Equipment maintenance or repair also plays an important role in the system. The person who maintains the equipment in the system is defined as the maintainer. The maintainer can open or close the equipment in accordance with the actual maintenance or repair; this ensures the safety of personnel during maintenance, in addition to confirming whether the maintenance is correct.

(b) Monitor operation: When the program starts, a polling mechanism will be used to monitor all the equipment, as shown in Fig. 9. Through RS-485 transmission, when it is determined that the connection is established, the program will read the status of each device. Once the device information is returned, the status of the device will be updated on the monitoring screen. If the device does not return relevant information, the status of the next device will be read. After reading the status, it will be determined whether the user has issued a command to change the device. If so, the status of the device will be changed immediately, or else, the status of the next device will be read. After receiving the setting command, the related settings will be set and transmitted to the device, as shown in the flowchart in Fig. 10.

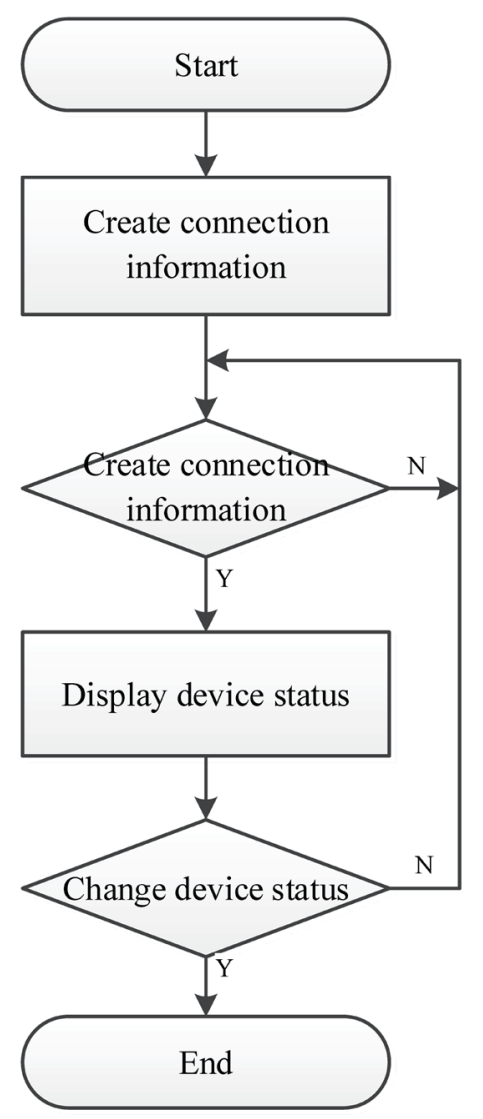

Fig. 9. Monitoring flowchart.

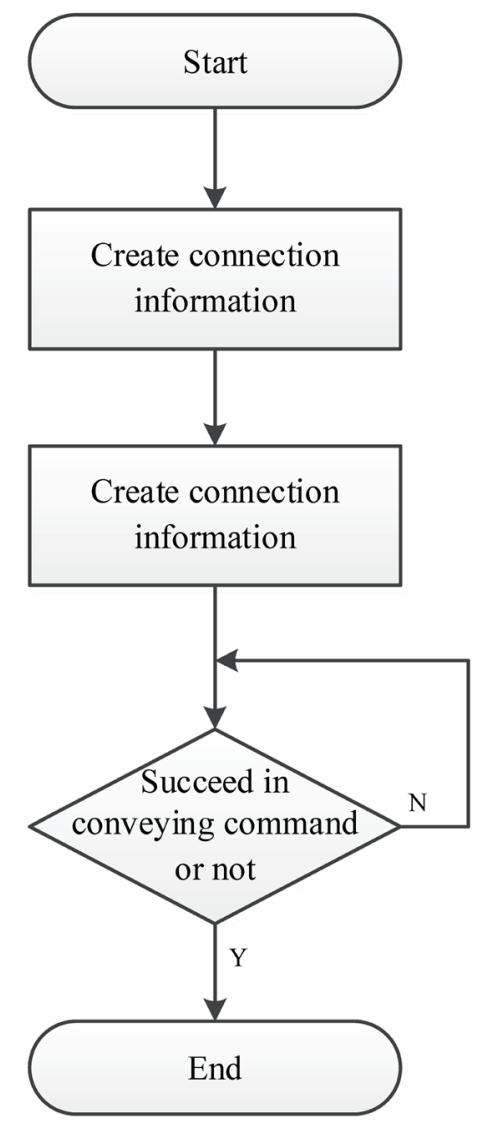

Fig. 10. Flowchart of monitoring the operation setting. 
However, unavailable setting may occur owing to a problem in the line communication or data processing of the equipment. If the problem persists, the program will abandon the setting process and notify the user. Equipment and monitoring management functions are planned for monitoring operations in response to the above monitoring operation functions. In the equipment management function, users can add, remove, and set devices managed by the system and the management parameters in accordance with the current status of the device. In the monitoring and management function, relevant information of the system device is provided, including the current status of the equipment and abnormal warnings, so that the maintenance personnel can take corresponding measures for specific situations.

(c) A report presents the quantity statistics of events and times, as well as report printing functions on the basis of the date range.

\section{Tests and Results}

The fabricated circuit board with capacitors for large-capacity power storage and the finished product of the Smart-CTD developed and produced in this study are shown in Figs. 11 and 12, respectively. The Smart-CTD has a sleek, practical appearance (with a cooling box design). Figure 13 shows the position of the high-voltage switchboard.

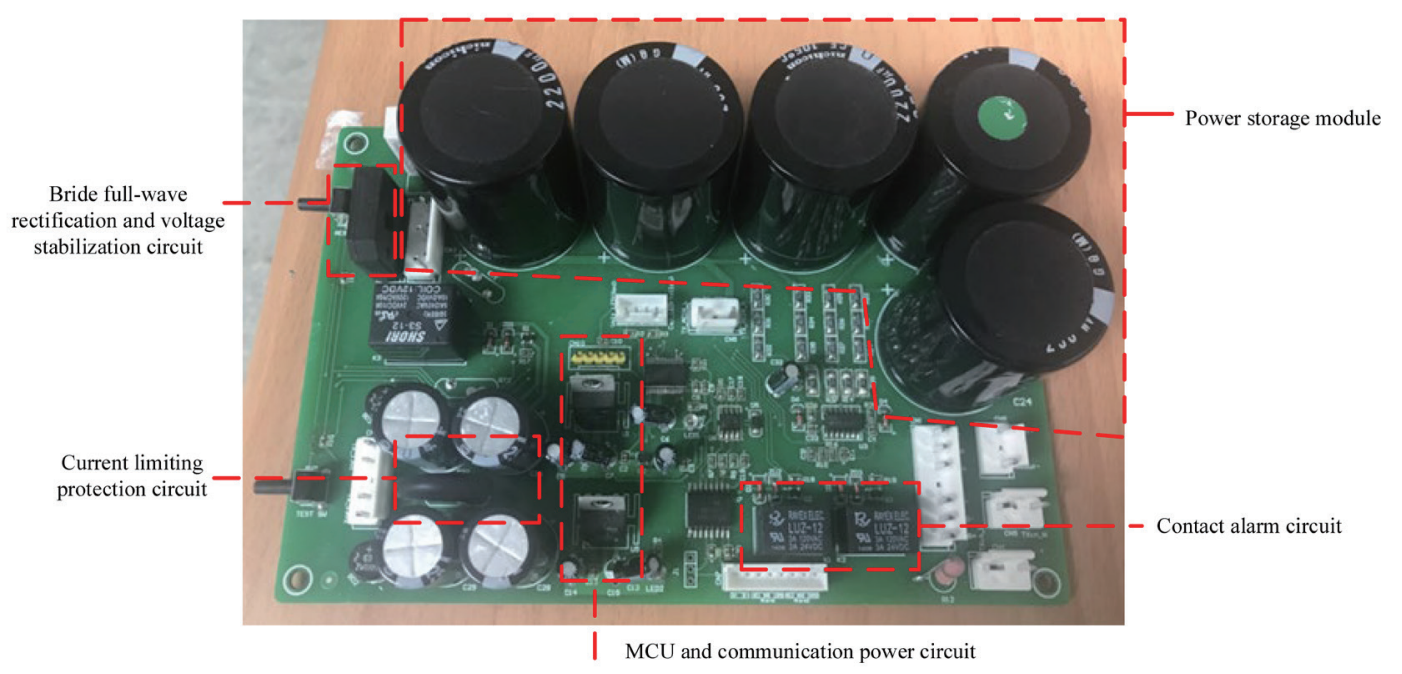

Fig. 11. (Color online) CTD circuit board.

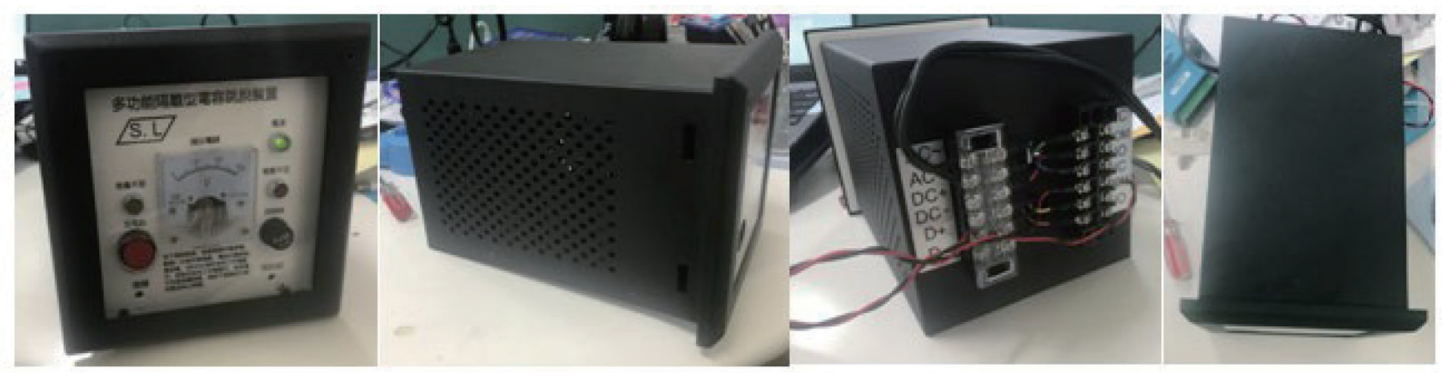

Fig. 12. (Color online) Appearance of Smart-CTD. 


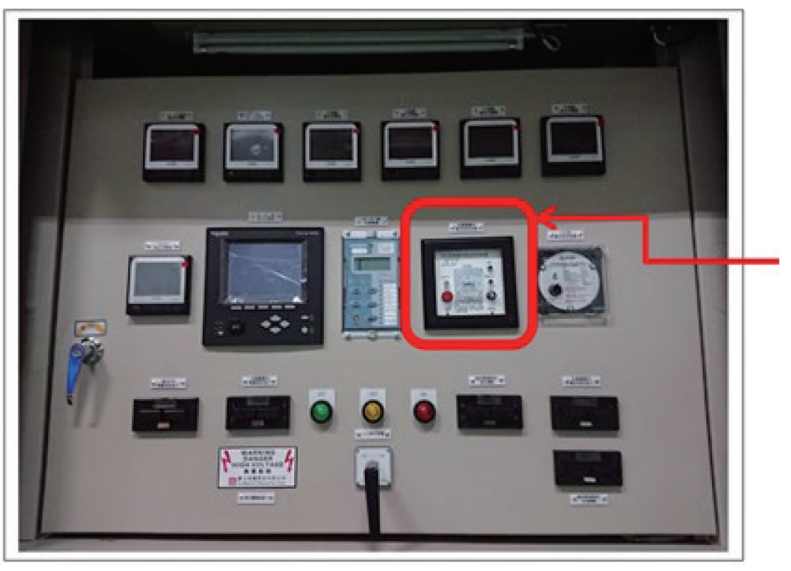

Fig. 13. (Color online) Smart-CTD position on highvoltage switchboard.

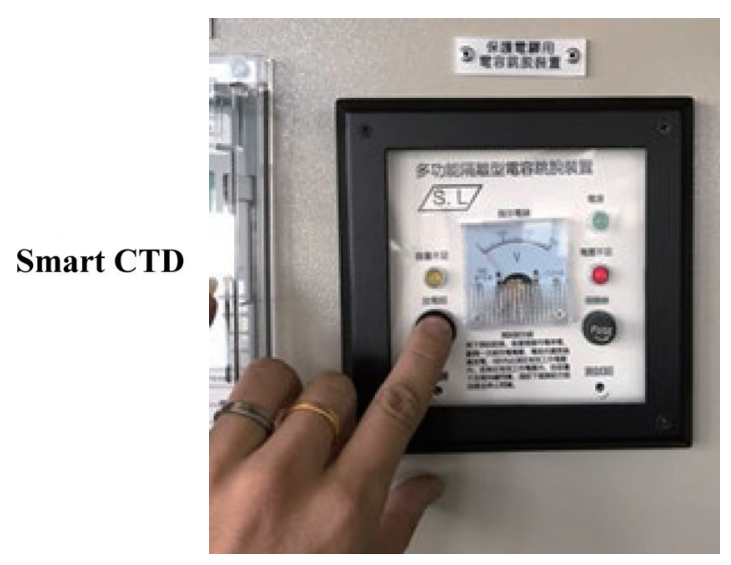

Fig. 14. (Color online) CTD discharge test.

The procedure of the Smart-CTD functional test is as follows.

\subsection{Test of DC power supply and discharge time of multifunctional CTD (AC input voltage $0 \mathrm{~V}$ )}

(1) Discharge time test of DC power supply without load

(a) When the voltage at the DC power supply terminal drops to $70 \%$ of its rated value, the time required for discharge is $223 \mathrm{~s}$.

(b) When the voltage at the DC power supply terminal drops to $50 \%$ of its rated value, the time required for discharge is $454 \mathrm{~s}$.

(c) When the voltage at the DC power supply terminal drops to $0 \%$ of its rated value, the time required for discharge is $2204 \mathrm{~s}$.

(2) Test when the DC power supply terminal is connected to the overcurrent protection relay with a load of $8 \mathrm{~W}$ : When the voltage at the DC power supply terminal drops to $70 \%$ of its rated value, the time required for discharge is $11.5 \mathrm{~s}$.

(3) Manual discharge test: Figure 14 shows the DC forced discharge test. When the user presses the discharge function key manually, the circuit of the high resistor inside the CTD will quickly discharge the DC power supply to $0 \mathrm{~V}$.

\subsection{Test for transmission and communication technology}

(1) The Modbus Scan communication test software was used to perform the RS-485 transmission communication test of CTD. The test software interface is shown in Fig. 15. Both Figs. 15(a) and 15(b) show the display for the test of the RS-485 transmission communication connection. 


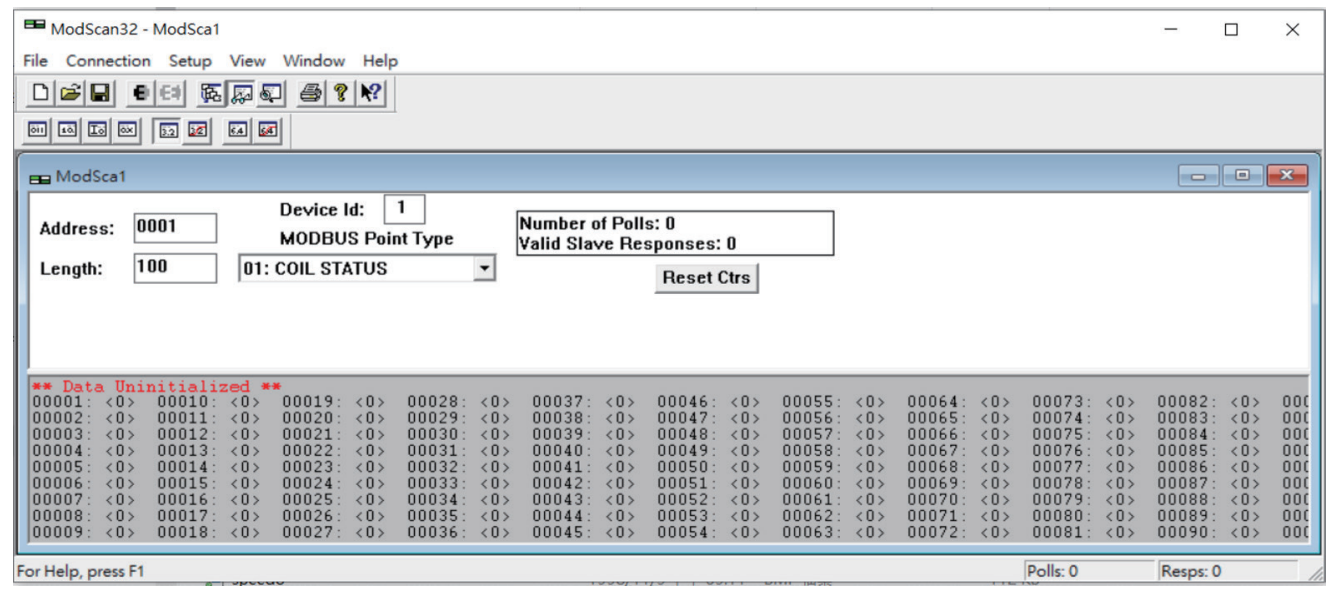

(a)

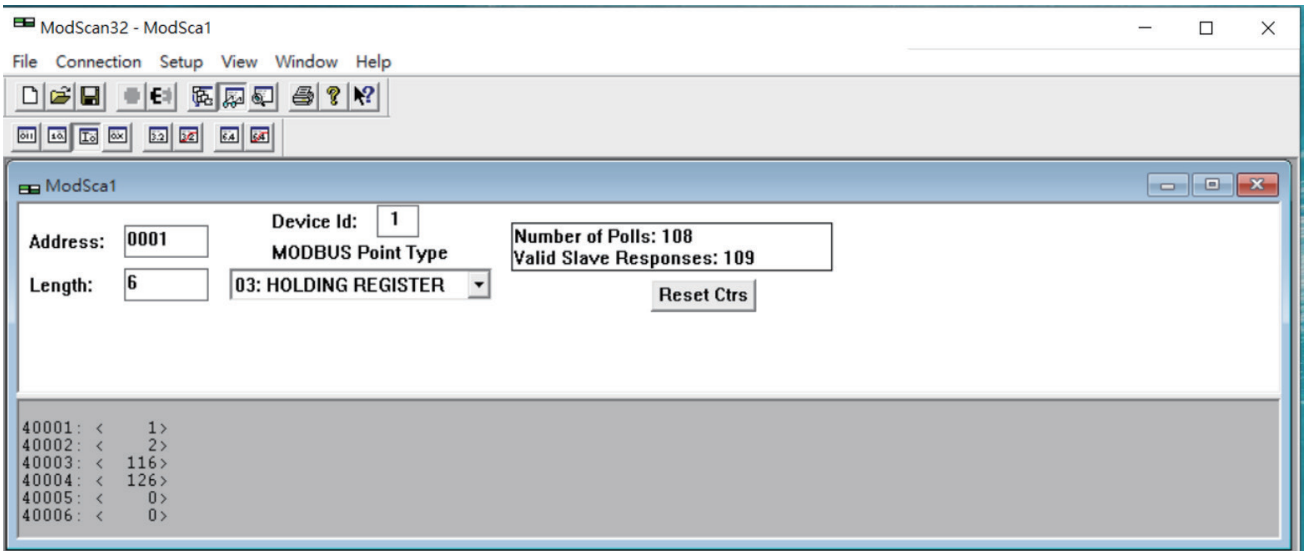

(b)

Fig. 15. (Color online) Modbus Scan communication test displays.

\subsection{Test and specifications of remote monitoring communication system}

(1) In this study, Microsoft Visual Studio C\# was used to develop remote monitoring and communication software. The program software design interface is shown in Fig. 16.

(2) The interface of Yuanrui monitoring and communication software is shown in Fig. 17. The operation interface is mainly designed to be convenient and easy for engineers. Users only need to select the ID of the Smart-CTD to collect data through RS-485 communication and upload the data to the server's database through the Internet. The server database interface is shown in Fig. 18.

The webpage programming interface of the webpage monitoring platform is shown in Fig. 19. The login interface of the web monitoring platform is shown in Fig. 20. The monitoring operation is shown in Fig. 21. Rights management is shown in Fig. 22. 


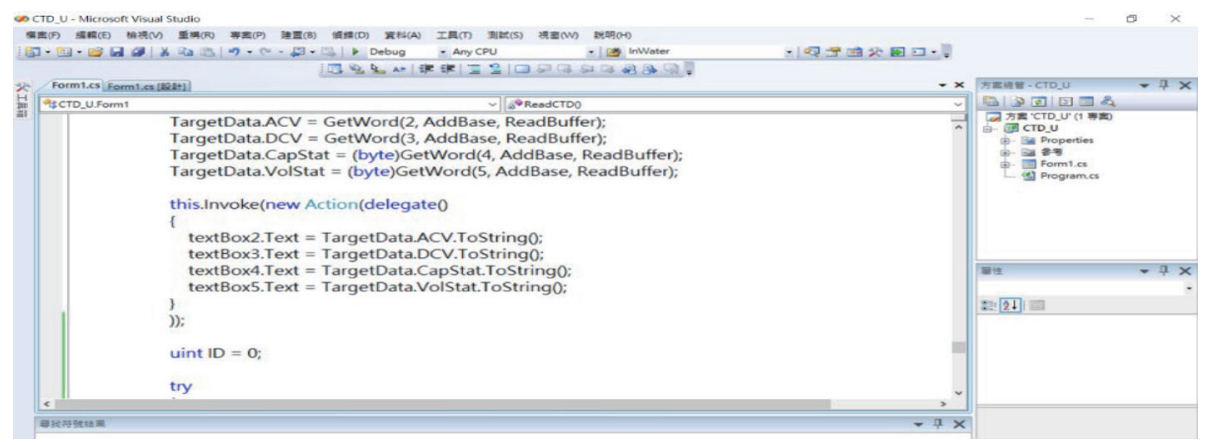

Fig. 16. (Color online) Interface of the software program.

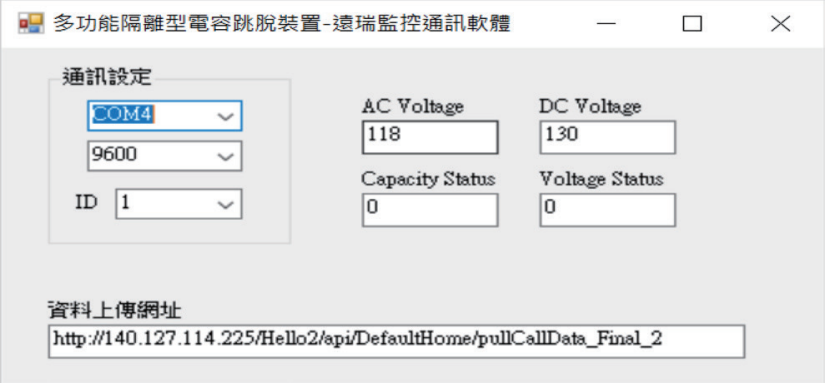

Fig. 17. (Color online) Interface of Yuanrui monitoring and communication software.

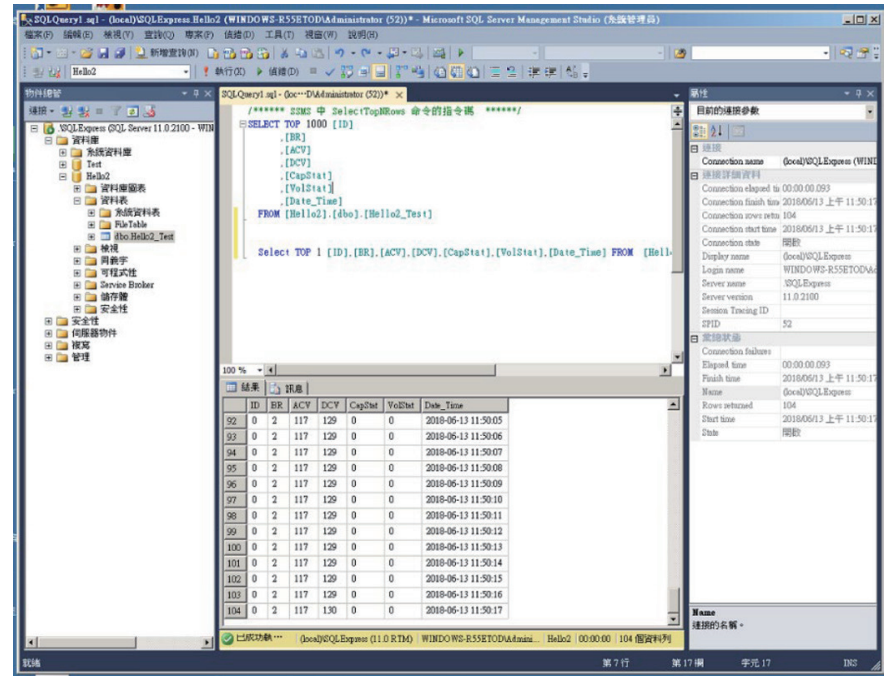

Fig. 18. (Color online) Interface of the server database.

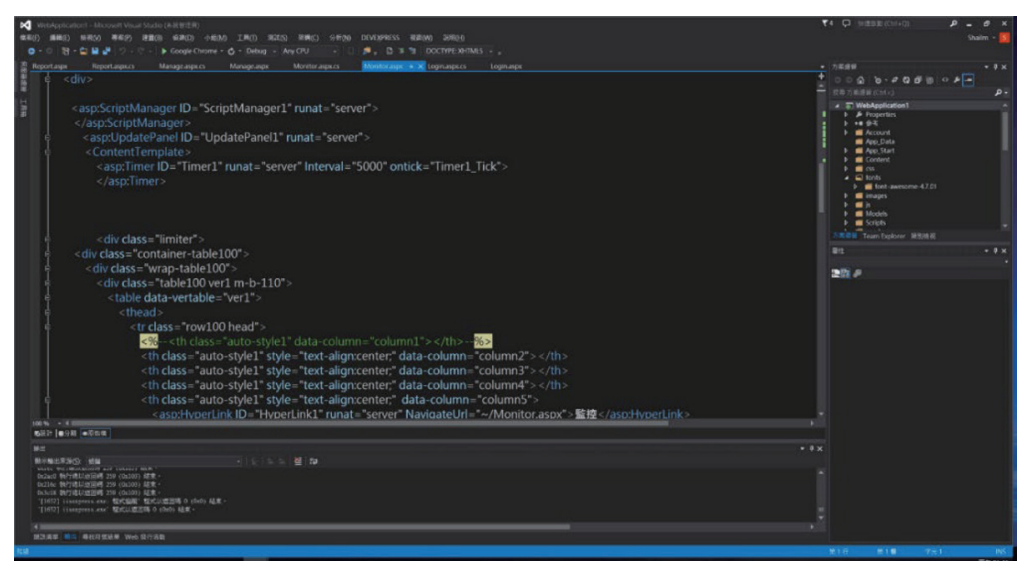

Fig. 19. (Color online) Interface of web programming. 


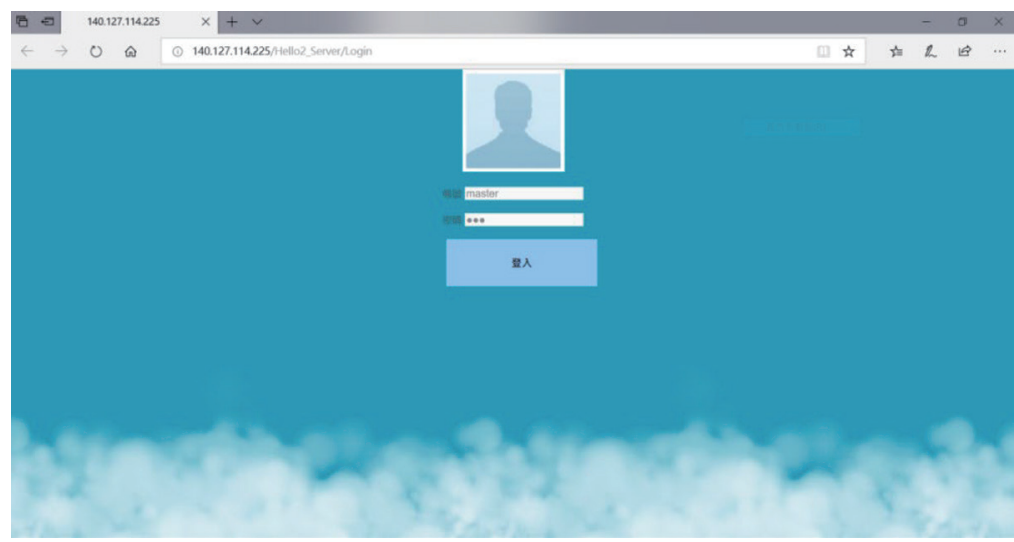

Fig. 20. (Color online) Login interface.

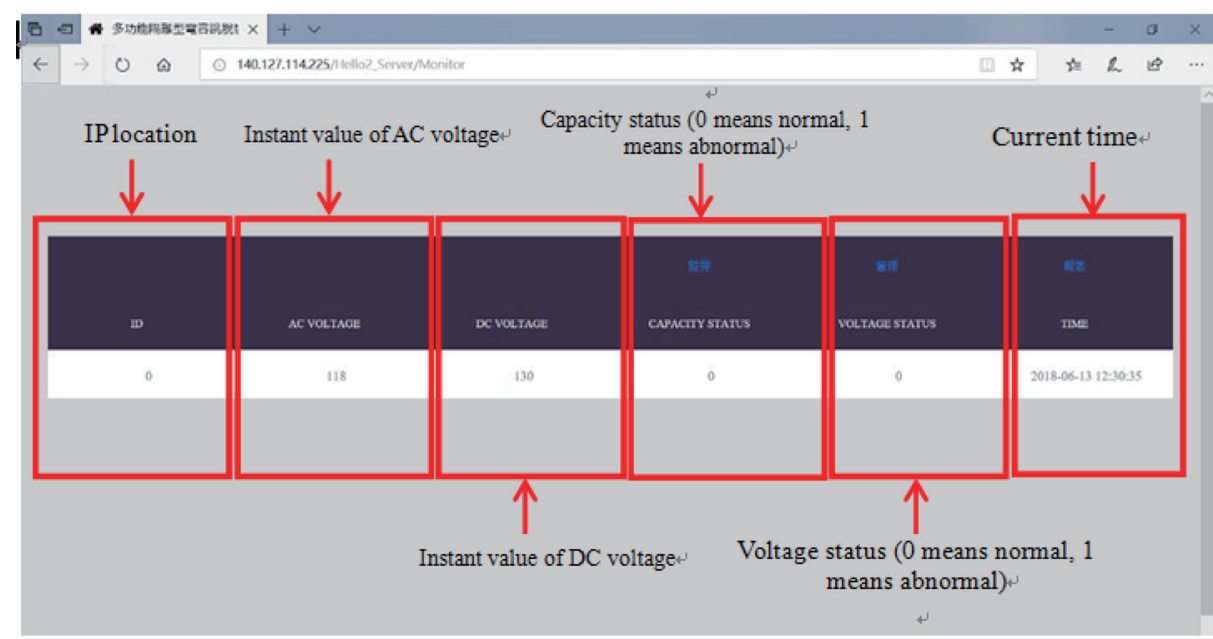

Fig. 21. (Color online) Interface of the monitor webpage.

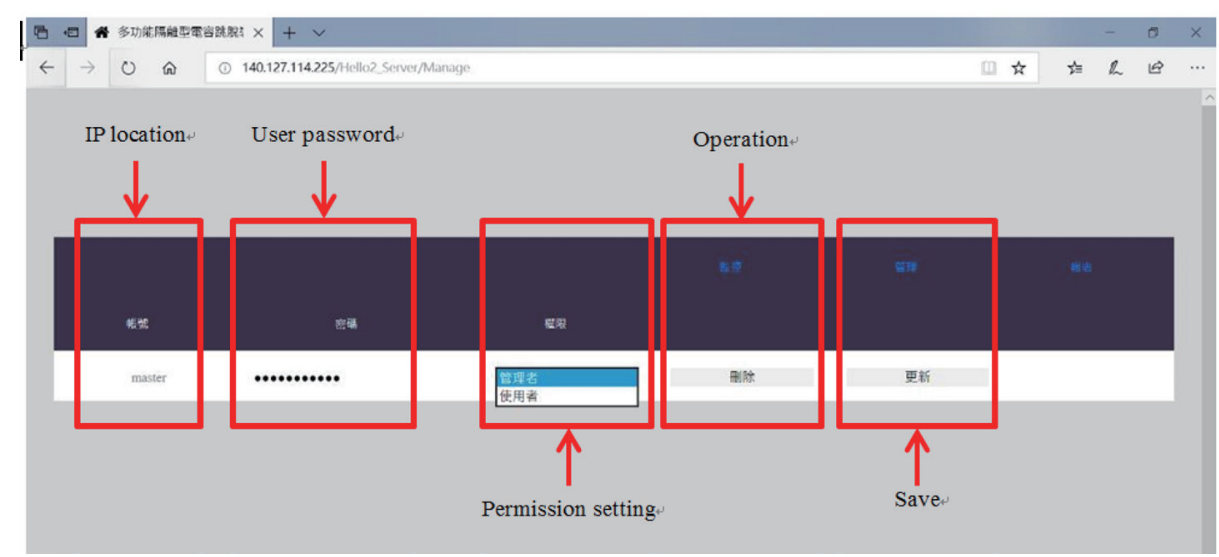

Fig. 22. (Color online) Interface of the rights management webpage. 


\section{Conclusions}

The protection relay of high-voltage users is a key device for tripping from power system failure to prevent the expansion of power accidents leading to damage to important power equipment. The CTD is a key device that maintains the normal operation of relay power to perform the tripping of the power circuit when the power supply of the system becomes abnormal. However, most traditional CTDs only use fuses for overcurrent disconnection protection. When a large current occurs upon fuse failure, belated current tripping and delayed alarm notification may easily result in appliance damage or disasters. Our Smart-CTD provides an isolated CTD to protect the circuit by limiting current and can automatically reduce the current when the operating temperature of the CTD device becomes very high, thereby protecting the equipment from being damaged by excessive heat caused by excessive current. When the temperature is very high and the current cannot be reduced any further, the circuit of the operating power will be disconnected to protect the back-end appliances from being burned. In addition, Smart-CTD has the RS-485 communication transmission and remote monitoring capability. Users can monitor the Smart-CTD installed in the power system in real time and synchronously be appraised of the status of all Smart-CTD devices in real time through the web monitoring platform. The output DC voltage charge-discharge test of the Smart-CTD and the network communication function test were performed in this study, and results met the design requirements.

\section{Acknowledgments}

The authors sincerely thank the Ministry of Science and Technology of Taiwan (MOST) and Southern Taiwan Science Park Bureau of Taiwan for supporting this research under Project numbers MOST 108-2622-E-992-027-CC3.

\section{References}

1 M. Aucoin, J. Zeigler, and B. Don Russell: IEEE Trans. Power Apparatus Syst. 104 (1985) 1455. https://doi. org/10.1109/MPER.1985.5526651

2 X. Tang, K. Kobayashi, Y. Sonobe, and M. Okazaki: 2011 Int. Conf. Advanced Power System Automation and Protection (2011) 210. https://doi.org/10.1109/APAP.2011.6180520

3 M. Parvania and M. Fotuhi-Firuzabad: IEEE Syst. J. 6 (2012) 35. https://doi.org/10.1109/JSYST.2011.2162877

4 H. Rudnick and L. A. Barroso: IEEE Power Energy Mag. 8 (2010) 18. https://doi.org/10.1109/MPE.2010.937465

5 L. Schmitt, J. Kumar, D. Sun, S. Kayal, and S. S. M. Venkata: IEEE Power Energy Mag. 11 (2013) 59. https:// doi.org/10.1109/MPE.2013.2258286

6 S.-J. Huang and H.-H. Wan: IEEE Trans. Energy Convers. 26 (2011) 987. https://doi.org/10.1109/ TEC.2011.2158022

7 S.-Y. Lu, L. Wang, S.-C. Ke, C.-H. Chang, and Zhi-hao Yang: IEEE Trans. Ind. Appl. 52 (2016) 42. https://doi. org/10.1109/IAS.2014.6978483

8 L.-Y. Chen, Y.-T. Yin, T.-Y. Ho, and Y.-Z. Chen: IEEE Nanotechnol. Mag. 8 (2014) 16. https://doi.org/10.1109/ MNANO.2014.2314182

9 C.-L. Kuo, J.-L. Chen, S.-J. Chen, C.-C. Kao, H.-T. Yau, and C.-H. Lin: IEEE Trans. Smart Grid 8 (2017) 1163. https://doi.org/10.1109/TSG.2015.2478855

10 S.-P. Lin, S.-K. Chang, H.-C. Lee, P.-T. Guo, S. Thiyagu, and C.-F. Lin: IEEE J. Photovoltaics 7 (2017) 184. https://doi.org/10.1109/JPHOTOV.2016.2621341 
11 C. Sena, R. Franco, and A. Giusto: IEEE PES General Meeting (2010) 1. https://doi.org/10.1109/ PES.2010.5590025

12 M. F. A. Sharkh, O. A. Sharkh, T. M. Alkhusaibi, and H. S. Alhadi: 2008 12th Int. Middle-East Power System Conf. (2008) 255. https://doi.org/10.1109/MEPCON.2008.4562357

13 J.-P. H. Knauss, C. Warren, and D. Kearns: PES T\&D 2012 (2012) 1. https://doi.org/10.1109/TDC.2012.6281507

14 H. B. Funmilayo, J. A. Silva, K. L. Butler-Purry: IEEE Trans. Power Delivery 27 (2012) 459. https://doi. org/10.1109/TPWRD.2012.2186181

15 L. Fendrick, T. Day, K. Fender, J. McCall, and A. Chaudhary: Conf. Record 2002 Annual Pulp and Paper Industry Technical Conf. (Cat. No.02CH37352) (2002) 194. https://doi.org/10.1109/PAPCON.2002.1015154

16 F. B. Ajaeiand and M. Sanaye-Pasand: 2008 Joint Int. Conf. Power System Technology and IEEE Power India Conf. (2008) 1. https://doi.org/10.1109/ICPST.2008.4745229

17 R. V. Jadhav, S. S. Lokhande, and V. N. Gohokar: 2016 Int. Conf. Computing Communication Control and Automation (ICCUBEA) (2016) 1. https://doi.org/10.1109/ICCUBEA.2016.7859994

18 M. Bagheri, A. Zollanvari, and S. Nezhivenko: IEEE Access 6 (2018) 9862. https://doi.org/10.1109/ ACCESS.2018.2809436

\section{About the Authors}

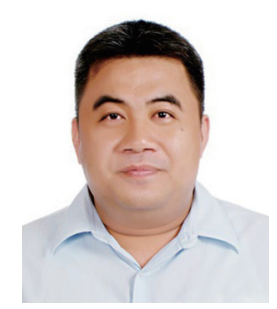

Cheng-Tsung Huang graduated with a master's degree in electrical engineering from National Kaohsiung University of Applied Science and Technology in 2012 and is currently working in the field of switchboards.

(bob@weilee-ee.com.tw)

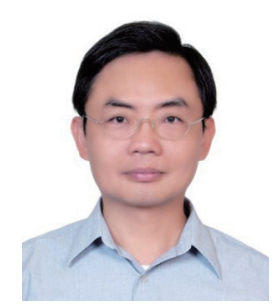

Ho-Chi Lien received his B.S. degree in 2004, his M.S. degree in 2007, and his Ph.D. degree in 2013, all from National Kaohsiung University of Applied Sciences, Taiwan, R.O.C., in civil and fire engineering. He is currently an assistant professor of the Fire Science Department, Tajen University. His current research interests include the applications of fire equipment and sensors and power design. (firemanlain@seed.net.tw)

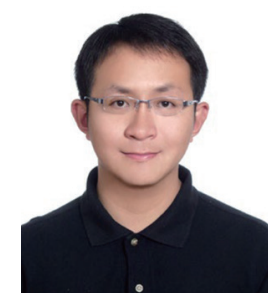

Chi-Pan Lin received his B.S. degree from National Kaohsiung University of Technology., Taiwan, R.O.C. He is skilled in graphics (AutoCAD secondgrade and hand-drawing third-grade licenses) and has worked in relevant fields of design, interior design of yachts, LED lighting design, and UPS cabinet design. (chipanlin@yahoo.com.tw)

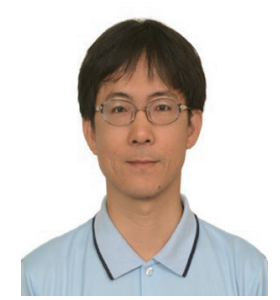

Wen-Ping Chen received his B.S. degree from National Taiwan Institute of Industrial Technology, Taiwan, R.O.C., in 1992, his M.S. degree from National Sun Yat-Sen University, Taiwan, R.O.C., in 2000, and his Ph.D. degree from National Kaohsiung University of Applied Sciences in 2008, all in electrical engineering. He is currently a professor of the Electrical Engineering Department, National Kaohsiung University of Science and Technology. His current research interests include the applications of Internet of Things and the protocol design of networks. (wpc@nkust.edu.tw) 